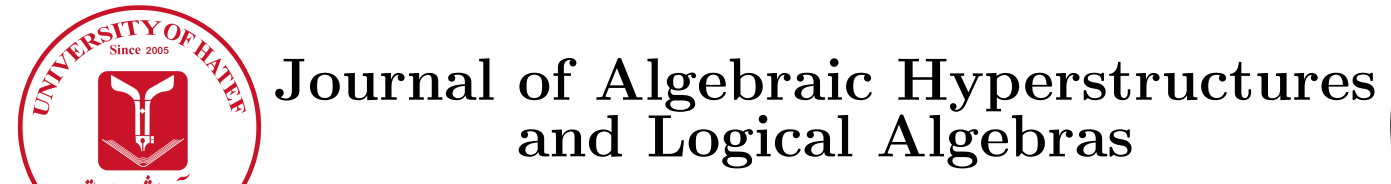

Special Issue (JAHA2020) Dedicated to Professor Piergiulio Corsini

Volume 1, Number 3, (2020), pp. 61-72

\title{
Hyper vector spaces over Krasner hyperfields
}

\author{
M. Al Tahan ${ }^{1}$ and B. Davvaz ${ }^{2}$ \\ ${ }^{1}$ Department of Mathematics, Lebanese International University, Lebanon \\ ${ }^{2}$ Department of Mathematics, Yazd University, Yazd, Iran \\ madeline.tahan@liu.edu.lb, davvaz@yazd.ac.ir
}

\begin{abstract}
In this paper, we study hyper vector spaces over Krasner hyperfields. First, we introduce the notions of linearly independence (dependence) and basis for a hyper vector space. Second, we investigate their properties and prove some results for hyper vector spaces that are similar to that of vector spaces over fields. Then, we define linear transformations over hyper vector spaces and investigate their properties. Finally, we prove the dimension theorem for linear transformations.
\end{abstract}

Article Information

Corresponding Author:

B. Davvaz;

Received: July 2020;

Accepted: August 2020.

Paper type: Original.

\section{Keywords:}

Krasner hyperfield, hyper vector space, subhyperspace, linearly independent, basis, linear transformation.

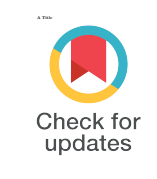

\section{Introduction}

Algebraic hyperstructures represent a natural generalization of classical algebraic structures and they were introduced by Marty [9] in 1934 at the eighth Congress of Scandinavian Mathematicians. Where he generalized the notion of a group to that of a hypergroup. A hypergroup is a non-empty set equipped with an associative hyperoperation and reproductive hyperoperation. In a group, the composition of two elements is an element whereas in a hypergroup, the composition of two elements is a non-empty set. Since then, many different kinds of hyperstructures (hyperring, hypermodule, hypervector space, ...) were widely studied from the theoretical point of view and for their applications to many subjects of pure and applied mathematics (see [3, 4, 5, 6, 11, 16, 17]). There exist different kinds of hyperrings. A special case of this type is the hyperring introduced by Krasner [8]. Also, Krasner introduced a new class of hyperrings and hyperfields: the quotient

https://doi.org/10.29252/HATEF.JAHLA.1.3.5 
hyperrings and hyperfields. For more details about Krasner hyperrings we refer to [6, 8, 12, Tallini in [14, 15] introduced the notion of hyper vector spaces over fields and studied basic properties of them. On the other hand, Vougiouklis [18] introduced weak hyper vector spaces, namely $H_{v^{-}}$ vector spaces. Later Ameri et al. in [1] studied the properties of dimension of hypervector spaces over fields and introduced the notions of linearly independent (respectively linearly dependent), generator and basis of a hypervector space.

The remainder part of this paper is organized as follows: In Section 2, we present some definitions related to hyperstructures that are used throughout the paper. In Section 3 we define hyper vector spaces over Krasner hyperfields and introduce some definitions like linearly independence, basis, .... Moreover, we investigate some properties of such spaces and prove that hyper vector spaces have similar properties to that of vector spaces. In Section 4, we define linear transformations between hyper vector spaces and introduce some definitions like the kernel, range, .... Also, we prove the Dimension theorem for linear transformations over hyper vector spaces.

\section{Basic definitions}

In this section, we present some definitions related to hyperstructures that are used throughout the paper (see [5, 6]).

Let $H$ be a non-empty set. Then, a mapping $\circ: H \times H \rightarrow \mathcal{P}^{*}(H)$ is called a binary hyperoperation on $H$, where $\mathcal{P}^{*}(H)$ is the family of all non-empty subsets of $H$. The couple $(H, \circ)$ is called a hypergroupoid. In this definition, if $A$ and $B$ are two non-empty subsets of $H$ and $x \in H$, then we define $A \circ B=\bigcup_{a \in A, b \in B} a \circ b, x \circ A=\{x\} \circ A$ and $A \circ x=A \circ\{x\}$. A hypergroupoid (H,०) is called: a semihypergroup if for every $x, y, z \in H$, we have $x \circ(y \circ z)=(x \circ y) \circ z$; a quasihypergroup if for every $x \in H, x \circ H=H=H \circ x$ (this condition is called the reproduction axiom); a hypergroup if it is a semihypergroup and a quasihypergroup. A Krasner hyperring is an algebraic structure $(R,+, \cdot)$ which satisfies the following axiom: $(1)(R,+)$ is a commutative hypergroup; (2) there exists $0 \in R$ such that $0+x=\{x\}$ for all $x \in R$; (3) for every $x \in R$ there exists unique $x^{\prime} \in R$ such that $0 \in x+x^{\prime} ;\left(x^{\prime}\right.$ is denoted by $\left.-x\right) ;(4) z \in x+y$ implies that $y \in-x+z$ and $x \in z-y$; (5) $(R, \cdot)$ is a semigroup having zero as a bilaterally absorbing element, i.e., $x \cdot 0=0 \cdot x=0$; (6) the multiplication "." is distributive with respect to the hyperoperation "+". Note that every ring is a Krasner hyperring. Different examples of Krasner hyperrings were constructed. We refer to [2, 5]. Let $(R,+, \cdot)$ be a Krasner hyperring and $A$ be a non-empty subset of $R$. Then $A$ is said to be a subhyperring of $R$ if $(A,+, \cdot)$ is itself a hyperring. A subhyperring $A$ of a Krasner hyperring $(R,+, \cdot)$ is a hyperideal of $R$ if $r \cdot a \in A(a \cdot r \in A)$ for all $a \in A, r \in R$. A commutative Krasner hyperring $(R,+, \cdot)$ with identity element "1" is a Krasner hyperfield if $(R \backslash\{0\}, \cdot)$ is a group.

\section{Dimension of hyper vector spaces}

In [1, Ameri et al. defined hyper vector spaces as an abelian group over a field and investigated their properties. In this section, we present a different definition for hyper vector spaces over Krasner hyperfields, study their properties and present an example applying the new defined notions.

Definition 3.1. Let $F$ be a Krasner hyperfield. A canonical hypergroup $(V,+)$ together with a map $\cdot: F \times V \rightarrow V$, is called a hyper vector space over $F$ if for all $a, b \in F$ and $x, y \in V$, the following conditions hold: (1) $a \cdot(x+y)=a \cdot x+a \cdot y$; (2) $(a+b) \cdot x=a \cdot x+b \cdot x$; (3) $a \cdot(b \cdot x)=(a b) \cdot x$; (4) $a \cdot(-x)=(-a) \cdot x=-(a \cdot x)$; (5) $x=1 \cdot x$. 
Definition 3.2. Let $F$ be a Krasner hyperfield. A canonical hypergroup $(V,+)$ together with a map : $F \times V \rightarrow V$, is called a weak hyper vector space over $F$ if for all $a, b \in F$ and $x, y \in V$, the following conditions hold: (1) $a \cdot(x+y) \subseteq a \cdot x+a \cdot y$; (2) $(a+b) \cdot x \subseteq a \cdot x+b \cdot x$; (3) $a \cdot(b \cdot x)=(a b) \cdot x$; (4) $a \cdot(-x)=(-a) \cdot x=-(a \cdot x)$; (5) $x=1 \cdot x$.

Example 3.3. Let $F$ be a Krasner hyperfield and $X$ be the set of all $n$-tuples with entries from $F$. Then, (1) $F$ is trivially a hyper vector space over itself; (2) $X$ is a weak hypervector space over $F$.

Next, we present a (non trivial) example of a hyper vector space.

Example 3.4. Let $(F,+, \cdot)$ be a Krasner hyperfield, $E$ be a non-empty set and $F^{E}$ be the set of all functions from $E$ to $F$. Then $\left(F^{E}, \oplus\right)$ is a hyper vector space over $F$. Where " $\oplus$ " and $\star: F \times F^{E} \longrightarrow F^{E}$ are defined as follows: For all $f, g \in F^{E}, k, x \in F$,

$$
(f \oplus g)(x)=f(x)+g(x) \text { and }(k \star f)(x)=k \cdot f(x) .
$$

Throughout this section, $F$ is a Krasner hyperfield, $V$ is a hyper vector space over $F$, " 0 " is the

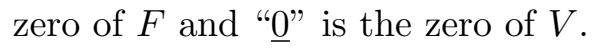

Definition 3.5. Let $F$ be a Krasner hyperfield and $(V,+)$ be a (weak) hyper vector space over $F$. $A$ non-empty subset $W \subseteq V$ is called subhyperspace of $V$ if for all $x, y \in W$ and $a \in F$, we have (1) $x-y \subseteq W$; (2) $a \cdot x \in W$.

Proposition 3.6. Let $F$ be a Krasner hyperfield and $(V,+)$ be a hyper vector space over $F$. If $a \in F$ and $x \in V$ then

(1) $-\underline{0}=\underline{0}$;

(2) $-(-a)=a$;

(3) $a \cdot \underline{0}=\underline{0}$;

(4) $0 \cdot x=\underline{0}$.

Proof. Let $x \in V$ and $a \in F$.

- Proof of 1 . Having that $-\underline{0}=-\underline{0}+\underline{0}$ and $\underline{0} \in-\underline{0}+\underline{0}$ implies that $-\underline{0}=\underline{0}$.

- Proof of 2. The proof follows from having $0 \in-a+a$ for all $a \in F$.

- Proof of 3. Since $-\underline{0}=\underline{0}$, it follows that $a \cdot \underline{0}=a \cdot(-\underline{0})=-a \cdot \underline{0}$. Having $\underline{0} \in a \cdot \underline{0}-a \cdot \underline{0}=$ $a \cdot(\underline{0}-\underline{0})=\{a \cdot \underline{0}\}$ implies that $a \cdot \underline{0}=\underline{0}$.

- Proof of 4 . Having $\underline{0} \in 0 \cdot x-0 \cdot x=(0-0) \cdot x=\{0 \cdot x\}$ implies that $0 \cdot x=\underline{0}$.

Proposition 3.7. Let $F$ be a Krasner hyperfield and $(V,+)$ be a hyper vector space over $F$. A non-empty subset $W \subseteq V$ is subhyperspace of $V$ if and only if $a \cdot x+b \cdot y \subseteq W$ for all $x, y \in W$ and $a, b \in F$.

Proof. Suppose that $W$ is a subhyperspace of $V$. Then $a \cdot x \in W$ and $-b \cdot y=(-b) \cdot y \in W$ for all $a, b \in F$. Thus, $a \cdot x+b \cdot y=a \cdot x-(-b \cdot y) \subseteq W$.

Conversely, suppose that $a \cdot x+b \cdot y \subseteq W$. By setting $b=0$, we get that $\{a \cdot x\}=a \cdot x+0 \cdot y \subseteq W$ and by setting $a=1, b=-1$, we get that $x-y \subseteq W$. Thus, $W$ is a subhyperspace of $V$. 
Definition 3.8. A subset $S=\left\{v_{1}, v_{2}, \cdots, v_{n}\right\}$ of a hypervector space $V$ over a Krasner hyperfield $F$ is called linearly independent if $c_{1}, c_{2}, \cdots, c_{n} \in F$ and $\underline{0} \in c_{1} \cdot v_{1}+c_{2} \cdot v_{2}+\cdots+c_{n} \cdot v_{n}$ then $c_{1}=c_{2}=\cdots=c_{n}=0$. A subset $S$ of $V$ is called linearly dependent if it is not linearly independent.

Remark 3.9. Let $S=\left\{v_{1}, v_{2}, \cdots, v_{n}\right\}$ be a subset of a hypervector space $V$ over a Krasner hyperfield $F$. Then, by Proposition 3.6; 4., $\underline{0} \in c_{1} \cdot v_{1}+c_{2} \cdot v_{2}+\cdots+c_{n} \cdot v_{n}$ has at least one solution; $c_{1}=\cdots=c_{n}=0$.

Definition 3.10. Let $S=\left\{v_{1}, v_{2}, \cdots, v_{n}\right\}$ be a subset of a hyper vector space $V$ over a Krasner hyperfield $F$ and $v \in V . v$ is said to be a linear combination of $S$ (or $v \in \operatorname{span}(S)$ ) if there exist $c_{1}, c_{2}, \cdots, c_{n} \in F$ such that $v \in c_{1} \cdot v_{1}+c_{2} \cdot v_{2}+\cdots+c_{n} \cdot v_{n}$.

In what follows and for simplicity, we write $a x$ instead of $a \cdot x$ for all $a \in F$ and $x \in V$.

Proposition 3.11. Let $S=\left\{v_{1}, v_{2}, \cdots, v_{n}\right\}$ be a subset of a hyper vector space $V$ over a Krasner hyperfield $F$. Then $S$ is linearly dependent if and only if one of its vectors is a linear combination of the others.

Proof. Suppose that $S=\left\{v_{1}, v_{2}, \cdots, v_{n}\right\}$ is linearly dependent. Then there exist $c_{1}, \cdots, c_{n} \in F$ such that $\underline{0} \in c_{1} v_{1}+\cdots+c_{n} v_{n}$ and at least one $c_{i} \neq 0$. We get now, by Proposition 3.6, that

$$
\underline{0}=c_{i}^{-1} \cdot \underline{0} \in d_{1} v_{1}+\cdots d_{i-1} v_{i-1}+v_{i}+d_{i+1} v_{i+1}+\cdots+d_{n} v_{n},
$$

where $d_{j}=c_{i}^{-1} c_{j}$ for all $j=1, \cdots, n$. The latter implies that there exists $x \in d_{1} v_{1}+$ $\cdots d_{i-1} v_{i-1}+d_{i+1} v_{i+1}+\cdots+d_{n} v_{n}$ such that $\underline{0} \in x+v_{i}$. Having $v_{i} \in-x+0=\{-x\}$ implies that $v_{i} \in-d_{1} v_{1}+\cdots-d_{i-1} v_{i-1}-d_{i+1} v_{i+1}+\cdots-d_{n} v_{n}$.

Conversely, without loss of generality, suppose that $v_{n} \in c_{1} \cdot v_{1}+\cdots+c_{n-1} \cdot v_{n-1}$. We have that $\underline{0} \in v_{n}-v_{n} \subseteq c_{1} v_{1}+\cdots+c_{n-1} v_{n-1}+(-1) v_{n}$. Thus, $S$ is linearly dependent.

Proposition 3.12. Let $V$ be a hyper vector space over a Krasner hyperfield $F$. Then

(1) a set with two vectors is linearly dependent if and only if one of them is a scalar multiple of the other

(2) a set containing $\underline{0}$ is linearly dependent;

(3) a set with one vector (that is not the zero vector) is linearly independent.

Proof. The proof of 1. follows from Proposition 3.11 .

- Proof of 2. This is clear by using Proposition 3.6 , as $\underline{0}=1 \underline{0}$.

- Proof of 3. Let $S=\{x\} \neq\{\underline{0}\}$ and $c \in F$. If $\underline{0} \in c x$ and $c \neq 0$ then $\underline{0}=c^{-1} \underline{0}=c^{-1}(c x)=$ $x$.

Definition 3.13. A subset $S=\left\{v_{1}, v_{2}, \cdots, v_{n}\right\}$ of a hyper vector space $V$ over a Krasner hyperfield $F$ is said to span $V$ if for every vector $v \in V$, there exist $c_{1}, c_{2}, \cdots, c_{n} \in F$ such that $v \in$ $c_{1} \cdot v_{1}+c_{2} \cdot v_{2}+\cdots+c_{n} \cdot v_{n}$.

Definition 3.14. A subset $S=\left\{v_{1}, v_{2}, \cdots, v_{n}\right\}$ of a hyper vector space $V$ over a Krasner hyperfield $F$ is said to basis for $V$ if it is linearly independent and it spans $V$. We say that $V$ is finite dimensional if it has a finite basis. Otherwise, it is called infinite dimensional. 
Proposition 3.15. Let $V$ be a hyper vector space over a Krasner hyperfield $F$ and $S=\left\{v_{1}, v_{2}, \cdots, v_{n}\right\}$ be a basis for $V$. Then every vector $v \in V$ is uniquely represented as linear combination of vectors in $S$.

Proof. Suppose that there exist $c_{i}, d_{i} \in F$ such that $v \in c_{1} v_{1}+\cdots+c_{n} v_{n}$ and $v \in d_{1} v_{1}+\cdots+d_{n} v_{n}$. Then $\underline{0} \in v-v \subseteq\left(c_{1}-d_{1}\right) v_{1}+\cdots+\left(c_{n}-d_{n}\right) v_{n}$. The latter implies that there exist $t_{i} \in c_{i}-d_{i}$ such that $\underline{0} \in t_{1} v_{1}+\cdots+t_{n} v_{n}$. Since $S$ is linearly independent, it follows that $t_{i}=0$ for all $i=1, \cdots, n$. We get now that $0 \in c_{i}-d_{i}$ for all $i=1, \cdots, n$. Having $(V,+)$ a canonical hypergroup implies that $c_{i}=d_{i}$ for all $i=1, \cdots, n$.

Theorem 3.16. Plus/Minus Theorem. Let $S$ be a non empty subset of a hyper vector space $V$ over a Krasner hyperfield $F$.

(1) If $S$ is linearly independent and $v \in V$ is not in span of $S$ then $S \cup\{v\}$ is linearly independent;

(2) If there exist a vector $v \in S$ such that $v$ is linear combination of other vectors in $S$ then $S-\{v\}$ and $S$ span the same space.

Proof. Let $S=\left\{v_{1}, \cdots, v_{n}\right\}$.

- Proof of 1. Let $\underline{0} \in c_{1} v_{1}+\cdots c_{n} v_{n}+c_{n+1} v$. Then $c_{n+1}=0$, otherwise $v \in \operatorname{span}(S)$. The latter implies that $\underline{0} \in c_{1} v_{1}+\cdots c_{n} v_{n}$. The linearly independence of $S$ asserts that $c_{1}=\cdots=c_{n}=0$.

- Proof of 2. Let $w \in \operatorname{span}(S)$. Then there exist $d_{1}, \cdots, d_{n} \in F$ such that $w \in d_{1} v_{1}+\cdots d_{n} v_{n}$. Without loss of generality, let $v_{n}$ be a linear combination of other vectors in $S$. Then there exist $c_{1}, \cdots, c_{n-1} \in F$ such that $w \in d_{1} v_{1}+\cdots+d_{n-1} v_{n-1}+d_{n}\left(c_{1} v_{1}+\cdots c_{n-1} v_{n-1}\right)=$ $\left(d_{1}+c_{1} d_{n}\right) v_{1}+\cdots+\left(d_{n-1}+c_{n-1} d_{n}\right) v_{n-1}$. The latter implies that $w \in \operatorname{span}\left(S-\left\{v_{n}\right\}\right)$.

Proposition 3.17. Let $V$ be a hyper vector space over a Krasner hyperfield $F, n \in \mathbb{N}$ and $W_{i}$ be a subhyperspace of $V$ for $i=1, \ldots, n$. Then

(1) $\{\underline{0}\}$ and $V$ are subhyperspaces of $V$;

(2) $W_{1} \cap W_{2}$ is a subhyperspace of $V$;

(3) $W_{1}+W_{2}=\left\{x+y: x \in W_{1}, y \in W_{2}\right\}$ is a subhyperspace of $V$;

(4) $\bigcap_{i=1}^{n} W_{i}$ is a subhyperspace of $V$;

(5) $\sum_{i=1}^{n} W_{i}$ is a subhyperspace of $V$.

Proof. The proof is straightforward.

Example 3.18. Let $F_{2}=\{0,1\}$ and define $\left(F_{2},+\right)$ and $\left(F_{2}, \cdot\right)$ by the following tables:

\begin{tabular}{|c|c|c|}
\hline+ & 0 & 1 \\
\hline 0 & 0 & 1 \\
\hline 1 & 1 & $F_{2}$ \\
\hline
\end{tabular}

\begin{tabular}{|l|l|l|}
\hline$\cdot$ & 0 & 1 \\
\hline 0 & 0 & 0 \\
\hline 1 & 0 & 1 \\
\hline
\end{tabular}


Define the map $\odot: F_{2} \times F_{2}^{3} \rightarrow F_{2}^{3}$ as:

$$
a \odot(x, y, z)=(a \cdot x, a \cdot y, a \cdot z)
$$

for all $a, x, y, z \in F_{2}$.

It is clear that $F_{2}^{3}$ is a weak hyper vector space over the Krasner hyperfield $F_{2}$. Define the subhyperspaces $W_{1}, W_{2}$ as

$$
W_{1}=\{(0,0,0),(0,1,0)\} \text { and } W_{2}=\{(0,0,0),(1,0,0),(0,0,1),(1,0,1)\} .
$$

One can easily see that $W_{1} \cup W_{2}=\{(0,0,0),(0,1,0),(1,0,0),(0,0,1),(1,0,1)\}$ is not a subhyperspace of $F_{2}^{3}$.

Proposition 3.19. Let $V$ be a (weak) hyper vector space over a Krasner hyperfield $F$ and $W_{1}, W_{2}$ be subhyperspaces of $V$. Then $W_{1} \cup W_{2}$ is a subhyperspace of $V$ if and only if $W_{1} \subseteq W_{2}$ or $W_{2} \subseteq W_{1}$.

Proof. If $W_{1} \subseteq W_{2}$ or $W_{2} \subseteq W_{1}$ then it is clear that $W_{1} \cup W_{2}$ is a subhyperspaces of $V$.

Let $W_{1} \cup W_{2}$ be a subhyperspaces of $V$. Suppose that $W_{1} \nsubseteq W_{2}$ and $W_{2} \nsubseteq W_{1}$. Then there are $x, y \in V$ such that $x \in W_{1}, y \in W_{2}, x$ is not in $W_{2}$ and $y$ not in $W_{1}$. Having $x+y \subseteq W_{1} \cup W_{2}$ implies that for all $z \in x+y$ we have $z \in W_{1} \cup W_{2}$. Without loss of generality, let $z \in W_{1}$. Having $z \in x+y$ implies that $y \in z-x \subseteq W_{1}$. The latter implies contradiction.

Definition 3.20. Let $V$ be a hyper vector space over a Krasner hyperfield $F$ and $S \subseteq V$. We define $L(S)$ as follows:

$$
L(S)=\left\{t \in V: t \in \sum_{i=1}^{n} a_{i} s_{i}, a_{i} \in F, s_{i} \in S, n \in \mathbb{N}\right\} .
$$

Proposition 3.21. Let $V$ be a hyper vector space over a Krasner hyperfield $F$ and $S \subseteq V$. Then $L(S)$ is the smallest subhyperspace of $V$ containing $S$.

Proof. Having $s=1 \cdot s \in L(S)$ for all $s \in S$ implies that $S \subseteq L(S)$.

Let $x, y \in L(S)$ and $a, b \in F$. Since $c \underline{0}=\underline{0}$ and $\underline{0}+x=x$ for all $c \in F, x \in V$ then we can write $a x+b y$ as follows:

$$
a x+b y=a \sum_{i=1}^{n} a_{i} s_{i}+b \sum_{i=1}^{m} b_{i} s_{i}=\sum_{i=1}^{\max (m, n)}\left(a a_{i}+b b_{i}\right) s_{i} \subseteq L(S) .
$$

Thus, $L(S)$ is a subhyperspace of $V$. Let $W$ be a subhyperspace of $V$ containing $S$. Then $\sum_{i=1}^{n} a_{i} s_{i} \subseteq W$ for all $s_{i} \in S \subseteq W$ and $a_{i} \in F$. Thus, $L(S) \subseteq W$.

Proposition 3.22. Let $V$ be a hyper vector space over a Krasner hyperfield $F$ and $W_{1}, W_{2}$ be subhyperspaces of $V$. Then $L\left(W_{1} \cup W_{2}\right)=W_{1}+W_{2}$.

Proof. Let $x \in W_{1} \cup W_{2}$. Then $x \in W_{1}$ or $x \in W_{2}$. Writing $x$ as $x=x+\underline{0}$ or $x=\underline{0}+x$ implies that $W_{1} \cup W_{2} \subseteq W_{1}+W_{2}$. Propositions 3.17 and 3.21 assert that $L\left(W_{1} \cup W_{2}\right) \subseteq W_{1}+W_{2}$. For every $z \in W_{1}+W_{2}, z \in x+y$ for some $x \in W_{1}, y \in W_{2}$. The latter implies that $x, y \in W_{1} \cup W_{2}$. We get now that $x+y \subseteq L\left(W_{1} \cup W_{2}\right)$. Thus, $W_{1}+W_{2} \subseteq L\left(W_{1} \cup W_{2}\right)$.

Proposition 3.23. Let $W$ be a non trivial subhyperspace of $V$ and $S$ be a spanning set for $W$. Then $S$ contains a linearly independent set that spans $W$. 
Proof. Let $S=\left\{v_{1}, \cdots, v_{n}\right\} \subseteq W$. Since every non zero vector in $V$ is linearly independent and $S$ spans $W \neq\{\underline{0}\}$, it follows that $S$ contains a linearly independent subset $S^{\prime}=\left\{v_{1}, \cdots v_{k}\right\}$. If $S$ is linearly independent, we are done. If not, remove the first $v_{j}$ such that $v_{j} \in \operatorname{span}(S)$. Using Plus/Minus Theorem, we get that $S$ and $S-\left\{v_{j}\right\}$ span $W$. If $S-\left\{v_{j}\right\}$ is linearly independent, we are done. If not, remove the first $v_{r}$ such that $v_{r} \in \operatorname{span}\left(S-\left\{v_{j}\right\}\right)$. Continuing on this pattern, we get the required result.

Corollary 3.24. Let $W$ be a subhyperspace of $V$ and $S$ be a spanning set for $W$. Then $S$ contains a basis for $W$.

Proof. Proposition 3.23 asserts that there exist a linearly independent subset of $S$ that spans $W$. Thus, $S$ contains a basis for $W$.

Theorem 3.25. Let $V$ be a hyper vector space of dimension $n$. Then every linearly independent subset of $V$ contains at most $n$ elements.

Proof. The proof is similar to that of Theorem 3.2 in [1].

Corollary 3.26. Let $B, B^{\prime}$ be two basis for a finite dimensional hyper vector space $V$. Then $|B|=\left|B^{\prime}\right|$.

Proof. Since $B$ is a basis for $V$ and $B^{\prime}$ is linearly independent subset of $V$, it follows by using Theorem 3.25 that $\left|B^{\prime}\right| \leq|B|$.

Since $B^{\prime}$ is a basis for $V$ and $B$ is linearly independent subset of $V$, it follows by using Theorem 3.25 that $|B| \leq\left|B^{\prime}\right|$.

Lemma 3.27. Let $V$ be a finite dimensional hyper vector space. Then every linearly independent subset $S$ of $V$ is contained in a finite basis of $V$.

Proof. Let $\operatorname{dim}(V)=n$ and $|S|=k$. Theorem 3.25 asserts that $k \leq n$. If $k=n$ then $S$ is a basis for $V$. Otherwise if $S$ is not a basis of $V$ then there exist $v_{1} \in V$ such that $v_{1}$ is not in $\operatorname{span}(S)$. Thus, by Plus/Minus Theorem, $S \cup\left\{v_{1}\right\}$ is linearly independent set. The latter contradicts Theorem 3.25. If $k<n$, then there exists $v_{1} \in V$ such that $v_{1}$ is not in $\operatorname{span}(S)$. By Plus/Minus Theorem, we get that $S \cup\left\{v_{1}\right\}$ is linearly independent. Again if $\left|S \cup\left\{v_{1}\right\}\right|=n$ then $S \cup\left\{v_{1}\right\}$ is a basis for $V$. Otherwise, $\left|S \cup\left\{v_{1}\right\}\right|=k+1<n$ and there exist $v_{2} \in V$ such that $v_{2}$ is not in $\operatorname{span}\left(S \cup\left\{v_{1}\right\}\right)$. Thus, by Plus/Minus Theorem, $S \cup\left\{v_{1}, v_{2}\right\}$ is linearly independent set. Continuing on this pattern, we get that $S \cup\left\{v_{1}, \cdots, v_{n-k}\right\}$ is a linearly independent set. Since $\left|S \cup\left\{v_{1}, \cdots, v_{n-k}\right\}\right|=n=\operatorname{dim}(V)$, it follows that $S \cup\left\{v_{1}, \cdots, v_{n-k}\right\}$ is a basis for $V$ containing $S$.

Lemma 3.28. Let $V$ be a finite dimensional hyper vector space and $W$ be a subhyperspace of $V$. Then dimension of $W \leq$ dimension of $V$.

Proof. Let $\operatorname{dim}(W)=k$. Then there exists a basis $S$ for $W$ such that $|S|=k$. Having $S$ a linearly independent set in $V$ implies, by Theorem 3.25 , that $k \leq \operatorname{dim}(V)$.

Let $V$ be a hyper vector space over $F$ and $W$ be a subhyperspace of $V$. Define $V / W=\{v+W$ : $v \in V$ with the hyperoperation: $(x+W) \oplus(y+W)=(x+y)+W$, and define $\star: F \times V / W \rightarrow V / W$ as follows: $a \star(x+W)=a \cdot x+W$.

Proposition 3.29. $V / W$ is a hyper vector space over $F$. 
Lemma 3.30. Let $V$ be a finite dimensional hyper vector space and $W$ be a subhyperspace of $V$. Then dimension of $V / W=$ dimension of $V$ - dimension of $W$.

Proof. Let $S=\left\{w_{1}, \cdots, w_{k}\right\}$ be a basis for $W$ and $\operatorname{dim}(V)=n$. Then, by Lemma 3.27, $S$ is contained in a basis $B=\left\{w_{1}, \cdots, w_{k}, v_{k+1}, \cdots, v_{n}\right\}$ for $V$. If $x \in V / W$ then there exists $v \in V$ such that $x=v+W$. Having $B$ a basis for $V$ and $v \in V$ implies that there exist $c_{1}, \cdots, c_{n}$ such that $x=v+W \in\left(c_{1} w_{1}+\cdots+c_{k} w_{k}+c_{k+1} v_{k+1}+\cdots+c_{n} v_{n}\right)+W=\left(c_{k+1} v_{k+1}\right)+$ $W+\cdots+\left(c_{n} v_{n}+W\right)=c_{k+1} \star\left(v_{k+1}+W\right)+\cdots+c_{n} \star\left(v_{n}+W\right)$. The latter implies that $\left\{v_{k+1}+W, \cdots, v_{n}+W\right\}$ spans $V / W$. To prove that $\left\{v_{k+1}+W, \cdots, v_{n}+W\right\}$ is linearly independent, let $\underline{0}+W \in c_{k+1} \star\left(v_{k+1}+W\right)+\cdots+c_{n} \star\left(v_{n}+W\right)=\left(c_{k+1} v_{k+1}+\cdots+c_{n} v_{n}\right)+W$. Then there exists $z \in W$ with $z \in c_{k+1} v_{k+1}+\cdots+c_{n} v_{n}$. Having $z \in W$ implies that there exist $d_{1}, \cdots, d_{k} \in F$ such that $z \in d_{1} w_{1}+\cdots+d_{k} w_{k}$. We get now $\underline{0} \in z-z \subseteq d_{1} w_{1}+\cdots+d_{k} w_{k}-c_{k+1} v_{k+1}-\cdots-c_{n} v_{n}$. Since $B$ is linearly independent, it follows that $d_{1}=\cdots=d_{k}=c_{k+1}=\cdots=c_{n}=0$.

Next, we present an example on a finite dimensional hyper vector space.

Example 3.31. Let $E=\{a, b\}, K=\{0,1,2\}$ and define $(K,+)$ and $(K, \cdot)$ by the following tables:

\begin{tabular}{|c|c|c|c|}
\hline+ & 0 & 1 & 2 \\
\hline 0 & 0 & 1 & 2 \\
\hline 1 & 1 & 1 & $K$ \\
\hline 2 & 2 & $K$ & 2 \\
\hline
\end{tabular}

\begin{tabular}{|l|l|l|l|}
\hline$\cdot$ & 0 & 1 & 2 \\
\hline 0 & 0 & 0 & 0 \\
\hline 1 & 0 & 1 & 2 \\
\hline 1 & 0 & 2 & 2 \\
\hline
\end{tabular}

Define $\underline{0}, f, g: E \longrightarrow K$ as follows:

$$
\underline{0}(a)=\underline{0}(b)=0, f(a)=0, f(b)=1, g(a)=1 \text { and } g(b)=0 \text {. }
$$

One can easily see the following:

(1) $K$ is a Krasner hyperfield,

(2) $K^{E}=\{\underline{0}, f, g, 2 f, f+g, 2 f+g, 2 g, f+2 g, 2 f+2 g\}$,

(3) $B=\{f, g\}$ is a basis for $K^{E}$,

(4) $W_{1}=\left\{\{\underline{0}, f, 2 f\}, W_{2}=\left\{\{\underline{0}, g, 2 g\}\right.\right.$ are non-trivial subhyperspaces of $K^{E}$ of dimension 1 .

\section{Linear transformations}

In this section, we define linear transformations between hyper vector spaces and investigate their properties. Moreover, we prove the Dimension theorem for linear transformations over hyper vector spaces.

Throughout this section, $F$ is a Krasner hyperfield, $U, V$ are hyper vector spaces, and we denote by "0" the zero of $F$, by " $\underline{0}$ " the zero of $U$ and by " $\overline{0}$ " the zero of $V$.

Definition 4.1. Let $U, V$ be two hyper vector spaces over a Krasner hyperfield $F$ and $T: U \rightarrow V$. Then $T$ is a linear transformation if for all $x, y \in U$ and $a \in F:(1) T(x+y)=T(x)+T(y)$; (2) $T(a x)=a T(x)$. 
Proposition 4.2. Let $U, V$ be two hyper vector spaces over a Krasner hyperfield $F$ and $T: U \rightarrow V$. Then $T$ is a linear transformation if and only if $T(a x+b y)=a T(x)+b T(y)$ for all $x, y \in U$ and $a, b \in F:$

Proof. The proof is straightforward.

Proposition 4.3. Let $T: U \rightarrow V$ be a linear transformation. Then for all $x \in U$ :

(1) $T(\underline{0})=T(\overline{0})$;

(2) $T(-x)=-T(x)$.

Proof. Let $x \in U$ and $y \in V$. Then, by Proposition $3.6,0 x=\underline{0}$ and $0 y=\overline{0}$.

- Proof of 1. $T(\underline{0})=T(0 x)=0 T(x)=\overline{0}$.

- Proof of 2 . Since $\underline{0} \in x-x$, it follows that $T(\underline{0}) \in T(x-x)=T(-x)+T(x)$. Using 1 ., we get that $\overline{0} \in T(-x)+T(x)$. Thus, $T(-x)=-T(x)$.

Example 4.4. Let $U, V$ be two hyper vector spaces over a Krasner hyperfield $F$. Then the following are linear transformations: (1) (Zero transformation) $T: U \rightarrow V$ defined by $T(x)=\overline{0}$ for all $x \in U$; (2) (Inclusion map) $T: U \rightarrow V$ defined by $T(x)=x$ for all $x \in U$. Here, $U$ is a subhyperspace of $V$; (3) $T: U \rightarrow V$ defined by $T(x)=k x$ for all $x \in U$ and a fixed $k \in F$. Here, $U$ is a subhyperspace of $V$.

Definition 4.5. Let $T: U \rightarrow V$ be a linear transformation. Then

(1) the kernel of $T$ is defined as: $\operatorname{ker}(T)=\{x \in U: T(x)=\overline{0}\}$;

(2) the range of $T$ is defined as: $R(T)=\{T(x): x \in U\}$.

Example 4.6. Let $T: V \rightarrow V / W$ defined by $T(x)=x+W$. Then $T$ is an onto linear transformation with $W$ as its kernel.

Let $T: U \rightarrow V$ be any transformation. Then, $T$ is one-to-one (1-1) if: $T(x)=T(y)$ implies $x=y ; T$ is onto if $R(T)=V ; T$ is an isomorphism if $T$ is linear, one to one and onto.

Proposition 4.7. Let $T: U \rightarrow V$ be a linear transformation. Then $T$ is one to one if and only if $\operatorname{ker}(T)=\{\underline{0}\}$.

Proof. Suppose that $T$ is one to one and let $x \in \operatorname{ker}(T)$. Using Proposition 4.3, we get that $T(x)=T(\underline{0})=\overline{0}$. Having $T$ one to one implies that $x=\underline{0}$.

For the converse, suppose that $\operatorname{ker}(T)=\{\underline{0}\}$ and let $x, y \in U$ such that $T(x)=T(y)$. Then $\overline{0} \in T(x)-T(y)=T(x-y)$. The latter implies that there exists $z \in x-y$ such that $T(z)=\overline{0}$. But $\operatorname{ker}(T)=\{\underline{0}\}$, then $z=\underline{0}$. Since $\underline{0} \in x-y$, it follows that $x=y$.

Proposition 4.8. Let $T: U \rightarrow V$ be a linear transformation. Then:

(1) $\operatorname{ker}(T)$ is a subhyperspace of $U$;

(2) $R(T)$ is a subhyperspace of $V$.

Proof. Suppose that $a, b \in F$. 
- Proof of 1. Let $x, y \in k e r(T)$. Then $T(a x+b y)=a T(x)+b T(y)=a \overline{0}+b \overline{0}=\overline{0}$. Thus, $a x+b y \subseteq \operatorname{ker}(T)$.

- Proof of 2. Let $v_{1}, v_{2} \in R(T)$. Then there exist $x, y \in U$ such that $T(x)=v_{1}, T(y)=v_{2}$. Since $T$ is a linear transformation, it follows that $a v_{1}+b v_{2}=a T(x)+b T(y)=T(a x+b y)$. The latter implies that for every $v \in a v_{1}+b v_{2}$, there exist $z \in a x+b y$ such that $T(z)=v$. Thus, $a v_{1}+b v_{2} \subseteq R(T)$.

Proposition 4.9. Let $T: U \rightarrow V$ be a linear transformation and $X, W$ be subhyperspaces of $U, V$ respectively. Then

(1) $T(X)=\{T(x): x \in U\}$ is a subhyperspace of $V$;

(2) $T^{-1}(W)=\{x \in U: T(x) \in W\}$ is a subhyperspace of $U$.

Proof. Let $a, b \in F$.

- Proof of 1. Let $T(x), T(y) \in T(X)$ for some $x, y \in X$. Then $a T(x)+b T(y)=T(a x+b y)$. Since $X$ is a subhyperspace of $U$, it follows that $a x+b y \subseteq X$. Thus, $a T(x)+b T(y) \subseteq T(X)$.

- Proof of 2. Let $x, y \in T^{-1}(W)$. The there exist $v, w \in W$ such that $T(x)=v, T(y)=w$. Since $a v+b w \subseteq W$, it follows that $T(a x+b y)=a v+b w \subseteq W$. Thus, $a x+b y \subseteq T^{-1}(W)$.

Proposition 4.10. Let $T: U \rightarrow V, J: V \rightarrow W$ be linear transformations. Then the composition of $J$ and $T, J \circ T$ is a linear transformation. Moreover, if $J, T$ are one to one then $J \circ T$ is one to one.

Proof. The proof is straightforward.

Proposition 4.11. Let $T: U \rightarrow V$ be an isomorphism. Then $T^{-1}$ is an isomorphism.

Proof. The proof is straightforward.

Proposition 4.12. Let $T: U \rightarrow V$ be a one to one linear transformation and $S \subseteq U$ be a linearly independent subset of $U$. Then $T(S)$ is a linearly independent subset of $V$.

Proof. Let $S=\left\{u_{1}, \cdots, u_{n}\right\}$ be a linearly independent subset of $U$. Suppose that $\overline{0} \in c_{1} T\left(u_{1}\right)+$ $\cdots+c_{n} T\left(u_{n}\right)$. Then $\overline{0} \in T\left(c_{1} u_{1}+\cdots+c_{n} u_{n}\right)$. The latter implies that there exist $z \in c_{1} u_{1}+\cdots+c_{n} u_{n}$ such that $T(z)=\overline{0}$. Having $T$ one to one implies that $z=\underline{0}$. We get now that $\underline{0} \in c_{1} u_{1}+\cdots+c_{n} u_{n}$. The independency of $S$ implies that $c_{1}=\cdots=c_{n}=0$.

Theorem 4.13. Dimension Theorem. Let $U$ be a finite dimensional hyper vector space and $T: U \rightarrow V$ be a linear transformation. Then

$$
\operatorname{dim}(\operatorname{ker}(T))+\operatorname{dim}(R(T))=\operatorname{dim}(U) .
$$

Proof. Let $B=\left\{u_{1}, \cdots, u_{n}\right\}$ be a basis for $U$. Since $\operatorname{ker}(T)$ is a subhyperspace of $U$, it follows that $\operatorname{dim}(\operatorname{ker}(T))=k \leq n$. We consider the following cases:

Case $k=0$. Since $\operatorname{ker}(T)=\{\underline{0}\}$ and $B$ is a basis for $U$, it follows by Proposition 4.12 that $T(B)=\left\{T\left(u_{1}\right), \cdots, T\left(u_{n}\right)\right\} \subseteq R(T)$ is linearly independent. Let $w \in R(T)$. Then there exists $u \in U$ such that $w=T(u) \subseteq T\left(c_{1} u_{1}+\cdots+c_{n} u_{n}\right)$ for some $c_{1}, \cdots, c_{n} \in F$. The latter implies that $w \in c_{1} T\left(u_{1}\right)+\cdots+c_{n} T\left(u_{n}\right)$. Thus $T(B)$ is a basis for $R(T)$ and hence, $\operatorname{dim}(R(T))=n$. 
Case $k=n$. Since $\operatorname{ker}(T)$ has a basis $S$ of $n$ linearly independent elements, it follows that $S$ is a basis for $U$. Having $T(S)=\overline{0}$ and $S$ a basis for $U$ imply that $T(U)=\overline{0}$. Thus, $\operatorname{dim}(R(T))=0$.

Case $0<k<n$. Let $S=\left\{s_{1}, \cdots . s_{k}\right\}$ be a basis for $\operatorname{ker}(T)$. By Lemma 3.27, $S$ is contained in a basis $B^{\prime}$ for $U$. Let $B^{\prime}=\left\{s_{1}, \cdots . s_{k}, w_{k+1}, \cdots, w_{n}\right\}$ and $v \in U$. Then there exist $c_{1}, \cdots, c_{n} \in F$ such that $v \in c_{1} s_{1}+\cdots+c_{k} s_{k}+c_{k+1} w_{k+1}+\cdots+c_{n} w_{n}$. Having $T$ a linear transformation implies that $T(v) \in T\left(c_{1} s_{1}+\cdots+c_{k} s_{k}+c_{k+1} w_{k+1}+\cdots+c_{n} w_{n}\right)=c_{1} \overline{0}+\cdots+c_{k} \overline{0}+c_{k+1} T\left(w_{k+1}\right)+\cdots+c_{n} T\left(w_{n}\right)$. We get that $T(v) \in c_{k+1} T\left(w_{k+1}\right)+\cdots+c_{n} T\left(w_{n}\right)$. Thus, $\left\{T\left(w_{k+1}\right), \cdots, T\left(w_{n}\right)\right\}$ spans $R(T)$. To prove that $\left\{T\left(w_{k+1}\right), \cdots, T\left(w_{n}\right)\right\}$ is linearly independent, let $\overline{0} \in c_{1} T\left(w_{k+1}\right)+\cdots+c_{n-k} T\left(w_{n}\right)$. Then $\overline{0} \in T\left(c_{1} w_{k+1}+\cdots+c_{n-k} w_{n}\right)$. The latter implies that there exists $u \in c_{1} w_{k+1}+\cdots+c_{n-k} w_{n}$ for some $c_{1}, \cdots, c_{n-k} \in F$ such that $T(u)=\overline{0}$. We get now that $u \in \operatorname{ker}(T)$. The latter implies $u \in$ $d_{1} s_{1}+\cdots+d_{k} s_{k}$ for some $d_{1}, \cdots, d_{k} \in F$. Since $\underline{0} \in u-u \subseteq d_{1} s_{1}+\cdots+d_{k} s_{k}-c_{1} w_{k+1}-\cdots-c_{n-k} w_{n}$ and having $B^{\prime}$ a basis for $U$, it follows that $c_{1}=\cdots=c_{n-k}=-d_{1}=\cdots=-d_{k}=0$. Thus, $\operatorname{dim}(R(T))=n-k$.

\section{Conclusion}

In this paper, we dealt with hyper vector spaces over Krasner hyperfields and linear transformations over them. We studied their properties, presented some results that are similar to that of vector spaces.

For future work, it will be interesting to introduce a norm over hyper vector spaces and study normed hyper vector spaces.

\section{References}

[1] R. Ameri, O.R. Dehghan, On dimension of hypervector spaces, European Journal of Pure and Applied Mathematics, 1(2) (2008), 32-50.

[2] M. Baker, N. Bowler, Matroids over hyperfields, (preprint). Available at: arxiv:math.CO/16 $01.0124 \mathrm{v} 5,2017$.

[3] P. Corsini, Prolegomena of hypergroup theory, Aviani Editore, (1993), 216 pp.

[4] P. Corsini, V. Leoreanu, Applications of hyperstructures theory, Advances in Mathematics, Kluwer Academic Publisher, 2003.

[5] B. Davvaz, Polygroup theory and related systems, World Scientific Publishing Co. Pte. Ltd., Hackensack, NJ, 2013. viii+200 pp.

[6] B. Davvaz, V. Leoreanu-Fotea, Hyperring theory and applications, International Academic Press, USA, 2007.

[7] W.C. Huffman, V. Pless, Fundamentals of error correcting codes, Cambrige, 2003.

[8] M. Krasner, A class of hyperrings and hyperfields, International Journal of Mathematics and Mathematical, 2 (1983), 307-312.

[9] F. Marty, Sur une generalization de la notion de group, In 8th Congress Mathematics Scandenaves, (1934), 45-49. 
[10] H.K. Mirdar, S.M. Anvariyeh, A hypervaluation of a hyperfield onto a totally ordered canonical hypergroup, Studia Scientiarum Mathematicarum Hungarica, 52(1) (2015), 87-101.

[11] S. Mirvakili, S.M. Anvariyeh, B. Davvaz, Transitivity of $\gamma$-relation on hyperfields, Bulletin Mathematique de la Societe des Sciences Roumanie (N.S.), 51(99) (2008), 233-243.

[12] S. Mirvakili, B. Davvaz, Relations on Krasner $(m, n)$-hyperrings, European Journal of Combinatorics, 31 (2010), 790-802.

[13] A. Nakassis, Expository and survey article of recent results in hyperring and hyperfield theory, International Journal of Mathematics and Mathematical, 11 (1988), 209-220.

[14] M.S. Tallini, Hypervector spaces, Proceedings of the Fourth International Congress on Algebraic Hyperstructures and Applications, Xanthi, Greece, (1990), 167-174.

[15] M.S. Tallini, Characterization of remarkable hypervector spaces, Proc. 8-th int. Congress on Algebraic Hyperstructures and Applications, Samotraki, Greece, (2002), Spanidis Press, Xanthi, (2003), 231-237.

[16] T. Vougiouklis, Hyperstructures and their representations, Aviani editor. Hadronic Press, Palm Harbor, USA, 1994.

[17] T. Vougiouklis, The fundamental relation in hyperrings. The general hyperfield, Proc. Fourth Int. Congress on Algebraic Hyperstructures and Applications (AHA 1990), World Scientific, (1991), 203-211.

[18] T. Vougiouklis, $H_{v}$-vector spaces, Algebraic hyperstructures and applications (Iasi, 1993), Hadronic Press, Palm Harbor, FL, (1994), 181-190. 